Revista de Ciencias Sociales - Número 68 (2016) - Páginas 37-62

La organización polifónica: una propuesta de negociación colaborativa...

\title{
LA ORGANIZACIÓN POLIFÓNICA: UNA PROPUESTA DE NEGOCIACIÓN COLABORATIVA BASADA EN LA TEORÍA DEL DISCURSO
}

\author{
THE POLYPHONIC ORGANIZATION: A PROPOSAL \\ FOR COLLABORATIVE NEGOTIATION \\ BASED ON DISCOURSE THEORY
}

\author{
MARÍA FERNANDA JUPPET EWING* \\ Universidad del Desarrollo (Chile) \\ mfjuppet@gmail.com
}

\section{Resumen}

El presente trabajo, busca proponer la aplicación de la teoría del discurso en las organizaciones polifónicas a través de la negociación colaborativa a la noción de empresa extendida, como estrategia para minimizar los conflictos sociales focalizados en proyectos económicos determinados en el marco de América Latina.

\section{Palabras clave}

Empresa extendida, organización polifónica, teoría del discurso, differend, traducción.

* LL.M. Universidad de California, Berkeley, MBA Universitat de Valencia, Profesora de Derecho, Economía y Mercado, Universidad del Desarrollo, Santiago, Chile. Artículo recibido el 10 de junio de 2016 y aceptado el 30 de junio de 2016.

Revista de Ciencias Sociales - Número 68 (2016) - Universidad de Valparáíso - ISSN 0716-7725-Valparáiso, Chile 


\section{Abstract}

The present work, looks to promote the aplication of the discursive theory in poliphonyc organizatios, through colavorative negotiation within the notion of extended organization, as a estrategy to minimize the social conflicts focused on especific economic projects in Latin America.

\section{Keywords}

Extended organization, pholiphonyc organization, theory of dicurse, differend, translation.

\section{Introducción}

Un importante desafío, que han debido comenzar a enfrentar las organizaciones que desean establecerse en América Latina, está dado por los conflictos sociales subyacentes ${ }^{1}$ que se manifiestan de manera directa en referencia a determinadas temáticas sociales y empresariales ${ }^{2}$.

Lo anterior, en el contexto de una sociedad de red, determinada por su velocidad, flexibilidad, maleabilidad y credibilidad ${ }^{3}$. Es particularmente a través de la característica de maleabilidad, que se demuestra la necesidad de adaptación a la que se someten las organizaciones empresariales en la actualidad, es lo que afecta la noción clásica de grupos de interés en la visión propuesta por el presente trabajo.

La visión tradicional de stakeholders o grupos de interés, es decir, entendiéndolos como los individuos, grupos y las organizaciones que

1. ALVES DE SOUZA, Filho: "La cuestión del sujeto individual y grupal en sociedades multiculturales en transformación". En: Revista Mexicana de Ciencias Políticas y Sociales, Vol. 50 N$^{\circ} 203$, 2008. Pág. 53 y ss.

2. PENAGLIA, Francisco y VALENZUELA VAN TREEK, Esteban: "Rebeldía en Calama: desafío al orden centralista chileno en un contexto de boom minero”. En: Revista Mexicana de Ciencias Políticas y Sociales,Vol. 59 N²22, 2014. Págs. 161 y ss

3. FRIEDMANN, Reinhard: "Gestión y organización de empresas en el siglo XXI”. LOM. Santiago. 2004.

Facultad de Derecho y Ciencias Sociales - Universidad de Valparaíso - Chile 
afectan la visión y misión de la compañía, se ven influidos por los resultados estratégicos alcanzados y tienen el derecho de exigir el buen desempeño de la empresa ${ }^{4}$, los circunscribe a ser comprendidos como parte de la metarealidad externa de la empresa.

Esta visión impide comprender hasta qué punto son actores claves dentro del proceso productivo de la organización ${ }^{5}$, parte de la propuesta de este artículo, es incorporarlos al interior de su análisis a través del concepto de empresa extendida ${ }^{6}$. Para la cual, los grupos de interés de una compañía no sólo influyen sus decisiones, sino que forman parte de su análisis estratégico, incorporando a administradores, accionistas, proveedores, consumidores, trabajadores y a la sociedad en su conjunto. De tal manera, surgen múltiples subculturas ${ }^{7}$ dentro de la organización, las cuales se caracterizan por tener visiones contrapuestas respecto de la actividad organizacional, particularmente el rumbo hacia el que debiera orientarse la compañía.

Estas visiones, dentro de sus diferencias generan, en los términos planteados por Lyotard, un differend, entendiendo por tal, una situación en la cual existe un conflicto irreconciliable entre las partes, que no puede resolverse equitativamente, porque ningún juicio de razón es aplicable simultáneamente para los planteamientos de los interesados ${ }^{8}$, que deben ser intervenidos por la administración, por una parte, por

4. KENNY, Graham: "From a Stakeholder Viewpoint: Designing measurable objectives”. En: Journal of Business Strategy. Vol. 33 N 6, 2012. Págs. 40 y ss.

5. ARDILES, JXXX: "De empresa y estrategia: Ideas y casos". En: Gráfica Metropolitana, Vol. XX N XX, 2012. Pág XXX.

6. HAX, Arnoldo y MAJLUF, Nicolás: "Lecciones en estrategia: Hacia una gestión de excelencia”. En: Ediciones UC. Santiago, 2014.

7. HOEFSTEDE, Geert: "Identifying organizational subcultures: An empirical approach”. En: Journal of management studies, Vol. 35 N $^{\circ}$, 1998. Págs. 1 y ss. Obtenido el 8 de junio de 2015 de: http://karhen.home.xs4all.nl/Papers/M\%26A/ Hofstede\%20(1998).pdf

8. LYOTARD, Jean Francois: “The Differend”. En: University of Minessota Press. Vol. 46, 1988. Pág. 13.

Revista de Ciencias Sociales - Número 68 (2016) - Universidad de Valparáíso - ISSN 0716-7725-Valparaíso, Chile 
medio de la interpretación, es decir, a través de la detección de las diferencias entre diversos lenguajes, con la finalidad de comprender el discurso del otro 9 , y, por otra, por la desarticulación del lenguaje, la que se caracteriza por ser una forma de intervención, mediante la cual se intenta cambiar las cosas a través de la intensificación máxima de una transformación en progreso ${ }^{10}$, para poder proyectar la supervivencia de la organización en el tiempo como meta principal ${ }^{11}$.

En el entendido que el lenguaje ha dejado de ser una simple herramienta que refleja la realidad, sino que se ha convertido en la clave para la comprensión de fenómenos sociales ${ }^{12}$, tales como las organizaciones empresariales. El presente trabajo se orienta a determinar si es que la propuesta de solución de controversias de la empresa polifónica, concibiéndola como una entidad de la que surgen múltiples discursos, muchas veces divergentes entre ellos ${ }^{13}$, basados en la multiculturalidad de los miembros de la organización ${ }^{14}$, expresado a través de la cultura organizacional, es decir, "la programación colectiva de la mente que diferencia a los miembros de una organización respecto de otra" ${ }^{15}$, pueden ser aplicadas a la noción de empresa extendida de

9. KORNBERGER, Martin, STEWART R., Clegg y CARTER, Chris: "Rethinking the polyphonic organization: Managing as discursive practice". En: Scadinavian Journal of Management, Vol: $22 \mathrm{~N}^{\circ} 1$, 2006. Págs. 3 y ss.

10. Ibíd, pág. 16.

11. DE GEUS, Arie y GORIN, Jorge: "La empresa viviente”. Ediciones Granica S.A. Buenos Aires. 2011.

12. CLEGG, Stewart, COUPASSON, David y PHILLIPS, Nelson: "Power and organizations”. En: Fundations for Organizational Science, Pine Forge Press, Londres, 2011.

13. HAZEN, Mary Ann: "Towards polyphonic organization". En: Journal of Organizational Change Management, Vol. $6 \mathrm{~N}^{\circ}$ 5, 1993. Págs. 15 y ss.

14. COX, Taylor y BLAKE, Stacy: "Managing cultural diversity: implications for organizational competitiveness”. En: The Executive. Vol. 3 N 5, 1991. Págs. 45 y ss. Obtenido el 13 de marzo de 2016 de: http://www.jstor.org/stable/4165021

15. HOEFSTEDE, Ibíd. página 2.

Facultad de Derecho y Ciencias Sociales - Universidad de Valparaíso - Chile 
Hax y Majluf, utilizando teoría del discurso como estrategia para morigerar los movimientos sociales que surgen en torno al desarrollo de sus actividades.

Asimismo, esta investigación busca hacer un planteamiento teórico que permita a las compañías distinguirse de sus competidores al lograr una relación directa y virtuosa con la comunidad a la cual afecta en el desempeño de su quehacer, alcanzando de esta forma su posicionamiento estratégico, lo que en términos porterianos implica que una compañía obtenga una ventaja competitiva sustentable al conseguir preservar aquello que se estima distintivo sobre dicha organización, esto significa actuar diferente respecto de los rivales o desarrollar actividades similares en formas distintas ${ }^{16}$.

El presente trabajo busca realizar un planteamiento diferenciador, en la forma en la que una organización inteligente pudiera desarrollar su proceso de evaluación de proyectos nuevos al lograr la licencia social de su entorno para operar a través de la teoría del discurso, partiendo del supuesto de que las organizaciones son sistemas verbales construidos socialmente ${ }^{17}$, y, por tanto, pueden ser intervenidos por sus miembros.

Este texto consta de seis partes: Primero, dentro del marco teórico, se analizan los nuevos paradigmas de la organización, comprendiendo la definición de empresa viviente ${ }^{18}$ y empresa extendida ${ }^{19}$, como un nuevo contexto a la teoría de las organizaciones, desde una perspectiva integral, reconociendo su capacidad de autopoiesis ${ }^{20}$, es decir, de auto

16. PORTER, Michael: "What is Strategy?”. En: Harvard Business Review, NovDic 1996. Págs. 61 y ss.

17. HAZEN. Ibíd.

18. DE GEUS. Ibíd.

19. HAX. Ibíd.

20. LIMONE, Aquiles: "La empresa viviente: Más allá de la metáfora”. Enefa. 2004. Arica. Chile. Obtenido de Escuela de Comercio Pontificia Universidad Católica de Valparaíso.

Revista de Ciencias Sociales - Número 68 (2016) - Universidad de Valparáíso - ISSN 0716-7725-Valparaíso, Chile 
crecimiento, evolucionando de manera autónoma en la medida de sus necesidades.

Segundo, y dentro del mismo marco, se estudia a la organización como sistema, comprendiendo que la conforman no sólo administradores, trabajadores y accionistas, sino que también proveedores, consumidores y la sociedad en su conjunto.

Tercero, se aborda el problema de los conflictos sociales, tanto individuales como multilaterale ${ }^{21}$, dentro del contexto social imperante en América Latina ${ }^{22}$, este acápite, nos provee de la trama necesaria para comprender los desafíos a los que se someten las empresas en la actualidad.

Cuarto, se realza la importancia de un diagnóstico temprano y una negociación rápida con la comunidad para evitar que un conflicto individual se convierta en un problema multilateral, dificultando sustancialmente su posibilidad de resolución.

Quinto, se analizan algunos aspectos fundamentales respecto de la teoría del discurso en el contexto de la organización polifónica ${ }^{23}$, como marco teórico para construir acciones colaborativas entre la empresa extendida y una de sus partes, particularmente la sociedad.

Sexto, se realiza una propuesta de solución de conflictos entre la organización y la comunidad a través de la teoría del discurso en la organización polifónica ${ }^{24}$, con miras a utilizar la negociación colaborativa como forma de estructura del diálogo ${ }^{25}$, con miras a la gestación de acuerdos que permitan a todos los involucrados un acercamiento real con el proyecto económico a desarrollar.

Con miras a revisar los temas propuestos, el problema en estudio será abordado desde una perspectiva teórica, mediante revisión bibliográfica de fuentes secundarias.

21. PENAGLIA. Ibíd.

22. ALVES DE SOUZA. Ibíd.

23. HAZEN. Ibíd.

24. KORNBERGER. Ibíd.

25. MAJLUF, Nicolás: "Los desafíos de la gestión: de lo formal a lo sutil”. Aguilar Ediciones. Santiago. 2011.

Facultad de Derecho y Ciencias Sociales - Universidad de Valparaíso - Chile 
La organización polifónica: una propuesta de negociación colaborativa...

\section{Los nuevos paradigmas de la organización}

Desde una visión tradicional que conceptualiza a la empresa es una organización que sólo refleja las decisiones de sus dueños, detalladamente determinada en el proceso de planificación estratégica ${ }^{26}$, se ha evolucionado hacia una perspectiva integral de las organizaciones, en las que se entiende la empresa como un ser viviente, superando la visión mecanicista, que la explica como una máquina guiada por sus gerentes, para comprender que ella crea su propio proceso, y por tanto, cambia y evoluciona en el tiempo ${ }^{27}$. Obligando a sus administradores a la gestación de un arquetipo dentro del cual encuadrar el crecimiento organizacional $^{28}$.

Asimismo, desde el planteamiento de las organizaciones inteligentes propuesta por Peter Senge, es posible caracterizarlas como entidades capaces de mejorar sus habilidades en forma consiente, para obtener los resultados que desea, surgiendo nuevos paradigmas desde el pensamiento colectivo ${ }^{29}$. De tal manera, se reconocen dos habilidades básicas en las organizaciones, la capacidad no sólo de tomar decisiones, sino que de desear de manera independiente a los individuos que las conforman. Y, en forma consecuencial, al comprender de esta manera la organización, podemos admitir que las empresas son capaces de aprender ${ }^{30}$.

26. DRUCKER, Peter. "La gerencia de Empresas”. Ediciones Sudamericana. Buenos Aires, 2012.

27. DE GEUS. Ibíd.

28. LIMONE, Aquiles y MARINOVIC, Milan: “The Theory of the Organization and the New Paradigms". En: Open Journal of Business and Management, 2013. Págs. 30 y ss.

29. SENGE, Peter, ROBERTS, Charlotte, ROSS, Richard, SMITH, Bryan, y KLEINER, Art: "La quinta disciplina en la práctica". Ediciones Granica S.A. Buenos Aires. 2012.

30. DE GEUS. Ibíd.

Revista de Ciencias Sociales - Número 68 (2016) - Universidad de Valparáíso - ISSN 0716-7725-Valparaíso, Chile 
Por tanto, ante la visión tradicional de comprensión de las organizaciones surge una postura que observa su quehacer desde una noción más amplia, influida por el entorno en el que se desenvuelve y las fuerzas de éste ${ }^{31}$. Los requerimientos de la empresa clásica se desdibujan, al comprender que la finalidad de la organización empresarial no está dada por la creación de bienes y servicios, sino que su razón de ser es el provocar satisfacción en las personas ${ }^{32}$.

En esta visión de la actividad empresarial, pasan a ser parte de la organización otros actores principales (accionistas, trabajadores, proveedores, clientes y sociedad), a los cuales debe satisfacer en su justa medida, para no quedar fuera del mercado ${ }^{33}$. En caso que el administrador no logre equilibrar todos estos intereses, arriesga seriamente la factibilidad de triunfo de la organización.

Las empresas se encuentran cada vez más conectadas con la sociedad, y cuando las bases del compromiso social comienzan a tambalear, la concepción de empresa también resiente este cambio ${ }^{34}$, es por esto que la capacidad de adaptación cobra vital importancia, basado en la comprensión del lenguaje de los stakeholders y sus expectativas ${ }^{35}$, pero desde una perspectiva integral, incorporándolos a la empresa misma, no sólo considerándolos como terceros interesados en los resultados de la organización.

De esta forma, para poder tomar decisiones estratégicas integradas, en las cuales la empresa no quede aislada del sistema del que forma parte, debe incorporarse en la dirección estratégica de la compañía esta visión "extendida" de la organización ${ }^{36}$. Así, la comunidad pasa a ser un co-creador de la organización ${ }^{37}$.

31. HAX. Ibíd.

32. ARDILES. Ibíd.

33. ARDILES. Ibíd.

34. MAJLUF. Ibíd.

35. FREEMAN, R. Edward y Mc VEA, John: "A Stakeholder Approach to Strategic Management". En: Working Paper $N^{o}$ 01-02: Virginia: Darden Graduate School of Business Administration, University of Virginia. 2001.

36. HAX. Ibíd.

37. FRIEDMANN. Ibíd.

Facultad de Derecho y Ciencias Sociales - Universidad de Valparaíso - Chile 
Los grupos de interés, es decir, los individuos, grupos y las organizaciones que influyen la visión y misión de la compañía, que se ven afectados por los resultados estratégicos alcanzados y tienen el derecho de exigir el buen desempeño de la empresa ${ }^{38}$. Son también conceptualizados como cualquier grupo o individuo que puede ser afectado por o afectar el logro de los objetivos de una organización ${ }^{39}$. Su importancia radica en los efectos que genera su renuncia a participar en la actividad empresarial, sin ellos no es posible realizar el proyecto estratégico de la organización ${ }^{40}$. La empresa no sólo tiene una dimensión económica, sino que social y política ${ }^{41}$, que debe considerarse dentro de su proyección en el tiempo y en la determinación de sus actividades.

\section{La organización como sistema}

Desde una perspectiva sistémica, cualquier actividad nueva que se desarrolle en un territorio determinado implica necesariamente consecuencias para la comunidad. Es importante ponderar aquellos efectos que pueden ser considerados como externalidades normales dentro de un proceso productivo pueden ser altamente invasivas para ésta, es decir, que reflexionen respecto del efecto del hombre en su entorno en las últimas décadas ${ }^{42}$.

$\mathrm{Al}$ crearse un solo sistema al interior de la empresa extendida, cada elemento forma parte del todo, sólo pudiendo comprenderse desde

\section{KENNY. Ibíd.}

39. FREEMAN. Ibíd.

40. HITT, Micahel, IRELAND, R. Duane y HOSKISSON, Robert: "Administración Estratégica: Competitividad y globalización: Conceptos y casos”. CENCAGE Learning. México. 2015.

41. CASTILLO, José: "Cambio social y enseñanza de administración de empresas”. En: Revista española de la opinión pública, N² 2, 1965. Págs. 109

y ss.

42. TINAS, Jorge: "La gestión ambiental del: ecosistema urbano". En: Ábaco, $\mathrm{N}^{\circ}$ 3, Otoño 1987. Págs. 12 y ss.

Revista de Ciencias Sociales - Número 68 (2016) - Universidad de Valparáíso - ISSN 0716-7725-Valparaíso, Chile 
una visión integral, en razón del lugar que ocupa dentro de la estructura de la que integra ${ }^{43}$, y, por tanto, participa en su propia definición, de tal manera no resulta posible el disociar la actividad productiva desplegada por una empresa del impacto que dicha actuación genera para la comunidad, al incluir a ambas dentro del mismo sistema complejo.

Una forma de mitigación de estas consecuencias se da a través de la autorregulación de la empresa. Al interior de la empresa, la regulación es una forma de imposición que en principio busca la eliminación de una conducta no deseada, pero que en definitiva la mantiene en el tiempo, dado que se produce un equilibrio entre el problema y la solución ${ }^{44}$.

Con la finalidad de minimizar las consecuencias no deseadas de las actuaciones de la organización, estas deben autorregularse, es decir, las normas surgen desde el interior de la organización, pero para determinar los preceptos de conducta que se orientarán a reestablecer el equilibrio entre la comunidad y la empresa se requiere la gestación de un diálogo fructífero que permita a las partes interpretar los planteamientos del otro, empatizando en las necesidades de cada una de las partes del sistema, para llegar a la implementación de soluciones que no impliquen un perjuicio ostensible para la comunidad.

De tal manera, la búsqueda de relaciones virtuosas entre la comunidad y la organización se convierte en un punto crucial de la estrategia empresarial, la sensibilidad con el entorno aparece como una de las características de las empresas longevas, dado que su capacidad de pertenencia con la comunidad les entrega una adaptabilidad que les permite subsistir en el tiempo ${ }^{45}$.

43. MIGUÉLES, Miguel Martínez: "Validez y confiabilidad en la metodología cualitativa”. En: Revista Paradigma. Vol. 27 N², 2014. Págs. 1 y ss.

44. BRAVO, Juan: "Análisis de Sistemas”. Evolución S.A. Santiago. 1998. Pág. 126

45. DE GEUS. Ibíd.

Facultad de Derecho y Ciencias Sociales - Universidad de Valparaíso - Chile 
La organización polifónica: una propuesta de negociación colaborativa...

\section{Conflictos sociales en América Latina y su relación con la empresa}

Así, en el desarrollo de un proyecto empresarial, se generarán necesariamente diferencias entre la organización y la comunidad que se siente afectada por éste, en ese momento el conflicto se encuentra en gestación. Podemos entender que estamos frente a un conflicto propiamente tal cuando diversas partes involucradas se dan cuenta de que existen diferencias que los separan al contraponer sus posturas, desarrollando un análisis racional de aquellos puntos ${ }^{46}$.

En un contexto social con una creciente muestra de descontento, particularmente enfocado en las organizaciones empresariales, tanto en el mundo como en Chile, podemos identificar causas ideológicas, autonómicas, o con base a problemas de integración, tales como clasificados por Penaglia y Valenzuela en "conflictos estudiantiles (2006, 2011,2013), mapuches (2010-2013) y conflictos territoriales-ambientales (Pascualama, 2005-2006 y 2013; Mehuin, 2006; Caimanes 2010; Castilla, 2010; Hidroaysén, 2011; Freirina 2012) $)^{47 ”, ~ g r a t u i d a d ~ d e ~ l a ~}$ educación (2014-2015), corrupción en la política (2015).

Dichos conflictos empresariales tienen su origen en fenómenos sociales que exceden el hito específico de la producción de bienes y servicios en el proyecto analizado, sino que, el tema relevante es la inclusión social o más bien la falta de inclusión social, usando una organización o grupo sectorial como elemento aglutinador de su molestia hacia el sistema en general. En el caso de los conflictos estudiantiles a las organizaciones de educación, Pascualama, Hidroaysén y Freirina a las empresas detrás de los proyectos empresariales, etc.

Si seguimos a Rodríguez y Arnold, es posible comprender que el problema de los fenómenos sociales que afectan a la empresa moderna tiene su origen en la exclusión de grupos sociales que manifiestan su imposibilidad de incorporarse a una realidad social que les es ajena mediante la expresión de sus necesidades urgentes de manera "peligrosa”.

\footnotetext{
46. MAJLUF. Ibíd.

47. PENAGLIA. Ibíd.
}

Revista de Ciencias Sociales - Número 68 (2016) - Universidad de Valparáíso - ISSN 0716-7725-Valparaíso, Chile 
Para estos autores, el principal desafío de la política pública en estos casos se encuadra en el tiempo de respuesta a las necesidades urgentes planteadas por los grupos excluidos, dado que la rapidez de respuesta demandada por los grupos sociales no tiene un correlato en el diseño, aplicación e implementación de la política pública ${ }^{48}$.

En una colectividad dinámica, los sujetos tanto individuales como grupales van asumiendo un activo rol en que rompen los cánones establecidos de la sociedad ${ }^{49}$. De tal manera, los actores sociales quieren formar parte de la toma de decisiones de las organizaciones relevantes para su comunidad, al comprender la empresa como una unidad extendida, es posible entender que las voces que se alzan desde ella, lo hacen también desde la organización, a través de sus trabajadores, consumidores y de la sociedad en su conjunto.

Hoy el sujeto siente que es capaz de cambiar su destino, formando parte integrante del proceso de transformación de la realidad que estima injusta $^{50}$. De la suma de estos individuos surgen grupos sociales, y de las alianzas entre grupos sociales los conflictos múltiples o rizomáticos, este contexto rodea a las empresas latinoamericanas. De tal manera, retoma validez el planteamiento de De Geus, cuando indica que las organizaciones están cada día más obligadas a adaptarse al contexto o desaparecer (De Geus, 2011).

Desde ese punto de vista, resulta importante comprender que la empresa es capaz de generar procesos de autopoiesis, es decir, autodefinir su identidad en forma autónoma (Limone, 2004). La reacción de las organizaciones frente a conflictos sociales hace imperioso que las compañías sean capaces de adaptarse a los nuevos requerimientos que la sociedad les realiza, con el riesgo de desaparecer si es que no son capaces de evolucionar a través del "gerentamiento del cambio interno" ${ }^{51}$.

48. RODRÍGUEZ, Darío y ARNOLD, Marcelo: "Sociedad y teoría de sistemas". Editorial Universitaria. Santiago. Chile 1991.

49. ALVES DE SOUZA. Ibíd.

50. ALVES DE SOUZA. Ibíd.

51. DE GEUS. Ibíd.

Facultad de Derecho y Ciencias Sociales - Universidad de Valparaíso - Chile 
Con todo, es importante indicar, que desde el momento en el cual el conflicto puntual de la empresa con su entorno se vuelve rizomático, aglutinando diversas causas a su alrededor, no es posible el diálogo con los interlocutores involucrados ${ }^{52}$, dado que se unen causas de tan diversa envergadura que no podrán ser solucionados los múltiples problemas involucrados por la organización sindicada como responsable.

De tal manera, resulta sustancial generar un diálogo temprano entre la comunidad inmediatamente afectada por la actividad empresarial y la organización, dentro del contexto de la empresa extendida, antes de que terceros ajenos al sistema logren un espacio dentro de la discusión, impidiendo cualquier posibilidad de diálogo e intervención directa desde el interior del proyecto.

\section{Problema de percepción}

Así, al comprender que la noción de empresa incorpora no sólo a administradores, dueños y trabajadores, integrando a proveedores, consumidores y la sociedad dentro de sus directrices estratégicas ${ }^{53}$, exige un cambio sustancial de la mentalidad de los directivos de las organizaciones. Siguiendo a De Geus, existe evidencia de que las corporaciones colapsan cuando los managers se identifican con el pensamiento y lenguaje de la economía, en vez de basarse en el de la colaboración entre los miembros de la organización ${ }^{54}$. Por tanto, resulta necesario el actuar con coherencia frente a los actores relevantes de la compañía, de tal manera de construir mediante el diálogo conjunto un proceso productivo que sea conscientemente aceptado por la comunidad, más allá de las mesas de diálogo en el contexto de autorizaciones medioambientales, dado que una vez que las confianzas se mellan, el capital social —entendiendo por tal, la credibilidad de la organización

\section{PENAGLIA. Ibíd. \\ 53. ARDILES. Ibíd. \\ 54. DE GEUS. Ibíd.}

Revista de Ciencias Sociales - Número 68 (2016) - Universidad de Valparáíso - ISSN 0716-7725-Valparaíso, Chile 
con su entorno-, con el que la organización cuenta para operar se reduce considerablemente.

Lo anterior es una consecuencia de un problema de apreciación, dado que cuando las personas se crean una percepción definida, resulta muy difícil modificarla, aun cuando se presente evidencia empírica que respalde la postura contraria a ella ${ }^{55}$. De tal manera, el momento ideal para intentar una intervención resulta previo a la gestación de dicha percepción preliminar, y mientras los actores involucrados en la diferencia son sólo aquellos directamente afectados. Es decir, antes de que el conflicto evolucione desde un conflicto individual a un conflicto multilateral o rizomático.

Pero, para lograr determinar una forma de acercamiento de las partes resulta necesario que dicha intervención se base en el espacio entre las diversas visiones de los miembros de la empresa extendida, justo en el lugar del differrend, entendiendo por tal, "una situación en la cual existe un conflicto irreconciliable entre las partes, que no puede resolverse equitativamente, porque ningún juicio de razón es aplicable simultáneamente para los planteamientos de ambas partes" ${ }^{\text {, }}$, dado que una intervención directa se percibe por los actores como un cuestionamiento a sus actuaciones por parte de la gerencia ${ }^{57}$, resulta necesario construir desde las diferencias sin el cuestionamiento de las válidas posturas de las voces internas de la organización que quieren manifestarse dentro del proceso de toma de decisiones ${ }^{58}$. Respetando con especial énfasis la multiculturalidad de los actores involucrados en el sistema organización ${ }^{59}$ al de la empresa extendida.

55. BARRET, Frank y COOPERRIDER, David: "Generative methaphor intervention: A new approach for working with systems divided by conflict and caught in defensive perception”. En: Journal of Applied Behavioral Sciene, Vol. 26 N², 1990. Págs. 219 y ss.

56. LYOTARD. Ibíd. Página 13.

57. BARRET. Ibíd.

58. HAZEN. Ibíd.

59. COX. Ibíd.

Facultad de Derecho y Ciencias Sociales - Universidad de Valparaíso - Chile 
Es imposible el aplicar soluciones estáticas para problemas dinámicos, dado que las soluciones deben ser capaces de aplicar una perspectiva distinta, ya que, si no es capaz de hacerlo, la supuesta solución sólo agravará el problema inicial ${ }^{60}$.

\section{La teoría del discurso y la polifonía en las organizaciones}

Desde la incorporación de modelos literarios para la comprensión de la realidad organizacional, surge el concepto de organización polifónica, al comprenderla como una entidad de la que surgen múltiples discursos ${ }^{61}$, muchas veces disonantes entre ellos, que tienden a ser reprimidos desde una visión tradicional de la administración ${ }^{62}$, surgidos desde la multiculturalidad de los miembros de la organización ${ }^{63}$.

Cada uno de los miembros de la organización se erige con una voz propia ${ }^{64}$, trayendo consigo su acervo cultural para el enriquecimiento de la entidad en la que se desempeña, que debe ser interpretada en sus diferencias, interviniendo los espacios de controversia que surgen en ellos, ya que es en esos espacios donde se esconde la capacidad de creación e innovación de la entidad ${ }^{65}$.

De tal manera, del espacio existente entre los diversos lenguajes ${ }^{66}$ que se presentan en las culturas subyacentes de la organización se esconden las potencialidades de creatividad de la organización ${ }^{67}$, que deben ser interpretados por los miembros del sistema ${ }^{68}$.

60. SENGE. Ibíd.

61. HOEFSTEDE. Ibíd.

62. HAZEN. Ibíd.

63. COX. Ibíd.

64. HAZEN. Ibíd.

65. KORNBERGER. Ibíd.

66. LYOTARD. Ibíd.

67. KORNBERGER. Ibíd.

68. CALLON, Michel: "Some elements of a sociology of translation: domestication of the scallops and the fishermen of St Brieuc Bay". En: The Sociological Review, VOL. 32 N $^{\circ} 1,1984$. Págs. 196 y ss.

Revista de Ciencias Sociales - Número 68 (2016) - Universidad de Valparáíso - ISSN 0716-7725-Valparaíso, Chile 
Así como en la organización resulta posible encontrar múltiples voces en su interior, de cuya intervención a través del diálogo surge la posibilidad de innovación ${ }^{69}$, no es menos cierto que la polifonía originalmente se refiere a la existencia de múltiples voces en relación a una idea ${ }^{70}$, por tanto, al aplicar la visión de la empresa extendida ${ }^{71}$, el sistema polifónico de la organización debe incorporar necesariamente a los stakeholders, como invitados externos de una meta realidad ${ }^{72}$.

El planteamiento de Hanzen al hacer comprensiva de la organización polifónica la descripción de Bakhin sobre las novelas de Dostoyevsky al caracterizarlas como "multivocales, intertextuales, con finales abiertos, cabeza abajo y casi caóticas"73, puede aplicarse plenamente para la empresa extendida, particularmente cuando se encuentra influida por movimientos sociales múltiples o rizomáticos, donde se generan alianzas temporales entre actores que se reúnen aparentemente en torno a un discurso común, pero que defienden causas independientes ${ }^{74}$.

Para referirse a la relación entre las organizaciones y las comunidades, Freire, en Hazen, plantea que el diálogo social se basa en relaciones mutuas y recíprocas, que son los pilares para el aprendizaje y la enseñanza de la libertad sobre la opresión ${ }^{75}$.

De tal manera, al comprender el lenguaje de los actores involucrados dentro de la empresa extendida, será posible realizar una intervención que permita interpretar ${ }^{76}$ las ideas de los actores presentes

69. HAZEN. Ibíd.

70. KORNBERGER. Ibíd.

71. HAX. Ibíd.

72. ARDILES. Ibíd.

73. HAZEN. Ibíd. Página 20.

74. PENAGLIA. Ibíd.

75. HAZEN. Ibíd. Página 23.

76. CALLON. Ibíd.

Facultad de Derecho y Ciencias Sociales - Universidad de Valparaíso - Chile 
dentro del differrend, desarticulando su discurso para reemplazarlo por un discurso común.

Comprendiendo que la sociedad forma parte integrante de la empresa extendida, debemos entender que sus discursos se comunican en diversos lenguajes, pero que deben ser interpretados en forma colaborativa para poder crear un lenguaje diferente que se erija como un nuevo paradigma que se aplique a ambas ${ }^{77}$.

Es importante recalcar que las voces consideradas "externas" a la organización por una visión tradicional, ignorando que el vínculo de influencia de la organización hacia la sociedad en la que está inserta es recíproco, olvida que esa comunidad en principio excluida forma parte de ella a través de sus colaboradores, por ende, las voces que intenta acallar no sólo se manifiestan fuera de la organización, sino que también en su interior. Por eso el valor de un individuo que plantea una perspectiva organizacional distinta es tan valiosa, porque en ella se crea un doble valor, en primer lugar, el espacio de innovación a través de la creatividad no aprovechada ${ }^{78}$, y, en segundo lugar, la posibilidad de comunicarse con el entorno al que necesita adaptarse para sobrevivir. De tal manera, la voz del entorno se encuentra escondida dentro de la cultura organizacional ${ }^{79}$.

Si comprendemos que la organización es un fenómeno social, y, que por su parte todo fenómeno social es un fenómeno lingüístico ${ }^{80}$, no podemos sino comprender que la organización construye realidad por medio de su práctica discursiva, y por tanto, cada voz individualmente considerada se convierte en una pieza fundamental del conflicto lingüístico del que surgirá la polifonía de la organización, sin aquel lenguaje individual no podríamos construir aquella.

\footnotetext{
77. KORNBERGER. Ibíd.

78. HAZEN. Ibíd.

79. HOEFSTEDE. Ibíd.

80. ECHEVERRÍA, Rafael: “Ontología del Lenguaje”. LOM Ediciones, Santiago. 2005 (Editor JC Sáez).
}

Revista de Ciencias Sociales - Número 68 (2016) - Universidad de Valparáíso - ISSN 0716-7725-Valparaíso, Chile 
El reconocer en el discurso de los movimientos sociales algún valor, desentrañar a través de una negociación colaborativa sus puntos de inflexión e intervenir dichas diferencias para adaptarse a los desafíos del entorno ${ }^{81}$ es lo que permitiría a las organizaciones inteligentes hacer frente a la nueva realidad de los movimientos sociales en América Latina. Esta solución sólo puede surgir desde la innovación más que de la noción de planificación estratégica ${ }^{82}$, entendiendo a la sociedad como parte misma del sistema complejo en que se ha convertido la organización empresarial.

La solución a este problema dista de lo trivial, al considerar que los proyectos empresariales involucran externalidades negativas que afectan a la comunidad, no existen los proyectos sin efectos externos de su implementación, incluso si es que estas consecuencias se minimicen al máximo, la organización se encuentra expuesta a fallas tecnológicas que generan consecuencias adversas respecto de la sociedad. La capacidad de contar con el tiempo suficiente para hacer frente a estos inconvenientes se sustenta en la credibilidad de la organización y el "capital social" con que se tenga al momento de presentarse un problema de estas características.

Resulta importante prevenir, que esta construcción no puede surgir sólo desde el diálogo, sino se requieren acciones significativas que refuercen dicho discurso para construir una nueva realidad social ${ }^{83}$.

\section{Una propuesta de solución a través de la organización polifónica}

Las empresas que desarrollan proyectos en América Latina, al intentar imponer sus planes de negocios amparados en un cumplimiento de la estricta normativa a que se encuentran sometidos sus procesos productivos, generaron una sensación de exclusión en las comunidades

81. MAJLUF. Ibíd.

82. HAMEL, Gary: "Strategy as revolution". En: Harvard Business Review. Obtenido el 8 de Junio de 2015 de: http://www.thegroupcreative.co.uk/wp-content/ uploads/Strategy-as-revolution-Gary-Hamel.pdf

83. CLEGG. Ibíd.

Facultad de Derecho y Ciencias Sociales - Universidad de Valparaíso - Chile 
adyacentes, que al no ser resuelta con premura se volvió peligrosa para sus objetivos ${ }^{84}$.

Por tanto, resulta necesario hacer frente a las discrepancias con el entorno antes de que la diferencia incorpore a movimientos sociales sin un interés directo en el proyecto empresarial, de ahí surge la urgencia de unir a la comunidad dentro de la empresa extendida, para solucionar el problema antes de que ella se haga pública y atraiga nuevos actores a la discusión.

Dada la importancia de la relación a largo plazo entre la comunidad y la empresa extendida que la alberga ${ }^{85}$, el proceso de deconstrucción del discurso entre ambos, y de intervención de sus desacuerdos ${ }^{86}$ debe desarrollarse a través de la negociación colaborativa, ya que debemos considerar que no sólo es importante el resultado que pueda alcanzar de una negociación, para que este proceso sea exitoso, se requiere que la forma en la que se logran los acuerdos sea eminentemente colaborativa, creando un espacio de cooperación entre los actores $^{87}$.

Es en este lugar de colaboración comienza la construcción del capital social de la compañía, que le permitirá desarrollar sus proyectos en armonía con la colectividad. Sólo tras la gestación de dicho capital social, la comunidad otorgará al final de las negociaciones una licencia social para operar a la organización, que le permita hacer frente a futuras fallas en sus procesos desde la confianza de la comunidad, en vez de al interior de un ambiente hostil. Evitando un conflicto social multilateral que le impida en la práctica desarrollar sus actividades.

No es necesario que las partes concuerden en todos los aspectos ${ }^{88}$, ni que se sientan conformes con todo el proceso, porque esto es imposible

\footnotetext{
84. RODRÍGUEZ. Ibíd.

85. DE GEUS. Ibíd.

86. KORNBERGER. Ibíd.

87. MAJLUF. Ibíd.

88. MAJLUF. Ibíd.
}

Revista de Ciencias Sociales - Número 68 (2016) - Universidad de Valparáíso - ISSN 0716-7725-Valparaíso, Chile 
ante el paradigma del differend ${ }^{89}$, sino que el éxito de esta negociación se basa en que su resultado sea lo mejor para la colectividad, y que refleje un proceso de colaboración entre los actores involucrados.

El ejercicio de negociaciones colaborativas que abran un espacio de trabajo conjunto para la comunidad y el resto de la empresa extendida, abre espacios de solidaridad que dotan a la empresa de sinergia, que conduce a "mayores niveles de orden en la organización"

De tal manera, la empresa, a través de su gobierno corporativo, debe anticipar los conflictos, siendo ésta una de las obligaciones de la organización $^{91}$, por medio de las conversaciones sostenidas entre los actores de la empresa extendida, estableciendo desde la negociación colectiva, parámetros más estrictos en el cumplimiento de obligaciones autoimpuestas, por medio del ejercicio de su libertad, autorregulando aquellas actuaciones que generan ineficacias del modelo afectando a la comunidad, entendiendo autorregulación como: “...la capacidad del mercado para encontrar sus propios equilibrios, sin injerencia alguna de los poderes públicos" ${ }^{\prime 2}$.

En igual sentido, existe un contenido superficial del discurso, dado por el lenguaje, y otro profundo, dado por las "reglas de la gramática" o el sentido en que comprendemos el lenguaje $e^{93}$. En los conflictos sociales que afectan el desarrollo de proyectos empresariales, el descontento con el sistema económico capitalista es un elemento que subyace $^{94}$, y que por tanto debemos considerarlo en el análisis a la hora

89. LYOTARD. Ibíd.

90. BRAVO. Ibíd. Página 151.

91. LIMONE. Ibíd.

92. GARDELLA, Ma Mercè DARNACULLETA. "Derecho administrativo y autorregulación: La autorregulación regulada”. Obtenido el 15 de junio de 2014 de: http://www.tdx.cat/bitstream/handle/10803/7681/tmdg.pdf? sequence $=7$ página 26.

93. CLEGG. Ibíd.

94. ALVES DE SOUZA. Ibíd.

Facultad de Derecho y Ciencias Sociales - Universidad de Valparaíso - Chile 
de generar una intervención en búsqueda de la licencia social para operar el proyecto determinado. $\mathrm{Al}$ comprender que tanto el lenguaje como las acciones significativas construyen realidad ${ }^{95}$, la permanencia en el tiempo de dicha licencia social tendrá como fundamento el cumplimiento de los acuerdos concordados por la cooperación entre la comunidad y la empresa.

Por tanto, y en resguardo de los acuerdos tomados con la comunidad, dentro de los puntos consignados en ellos, se debe incorporar que cada uno de los términos convenidos por las partes tienen un significado que es válido para cada una de ellas ${ }^{96}$, y que debe, por tanto, ser conciliado al momento de la negociación colaborativa. Reconociendo el lenguaje común que regirá a las partes involucradas en el consenso.

Con todo, esta confianza es recíproca, dado que, si es que la empresa no se encuentra conforme con los actos de la comunidad, sólo queda a la empresa cerrar el proyecto ${ }^{97}$, afectando en forma directa el empleo de los habitantes de la zona, y, en forma consecuencial, la calidad de vida de la comunidad en su conjunto.

\section{Conclusiones}

Las empresas, como cualquier organización, están en la necesidad de adaptarse para sobrevivir en el tiempo ${ }^{98}$. Sin este cambio de paradigma ${ }^{99}$ no serán capaces de hacer frente al cambio de percepción de sus grupos de interés ${ }^{100}$.

95. CLEGG. Ibíd.

96. CLEGG. Ibíd.

97. RADIO COOPERATIVA: "Agrosuper anunció el cierre indefinido de la planta en Freirina” 10 de de Diciembre de 2012. Obtenido el 24 de julio de 2015 de: http://www.cooperativa.cl/noticias/pais/region-de-atacama/conflicto-en-freirina/ agrosuper-anuncio-el-cierre-indefinido-de-la-planta-en-freirina/2012-12-10/ 174949.html
98. DE GEUS. Ibíd.
99. LIMONE. Ibíd.
100. FREEMAN. Ibíd.

Revista de Ciencias Sociales - Número 68 (2016) - Universidad de Valparáíso - ISSN 0716-7725-Valparaíso, Chile 
Al considerar la definición de organización desde la noción de empresa extendida ${ }^{101}$, que incorpora a los diversos grupos de interés en la metarealidad que determina el sentido de la organización ${ }^{102}$, se minimizan los riesgos de resistencia desde la sociedad hacia el proyecto económico que se busca desarrollar por la entidad.

Asimismo, desde la noción de organización polifónica ${ }^{103}$, las voces disonantes de la comunidad pasan a ser subculturas dentro de la cultura organizacional ${ }^{104}$, escondiendo potencialidades de creatividad e innovación dentro de su lenguaje ${ }^{105}$, al incorporar la noción de empresa extendida, admite el ampliar las voces a ser escuchadas dentro de la organización.

Utilizando teoría del discurso para poder intervenir las diferencias entre los lenguajes de los grupos de interés afectados por la organización ${ }^{106}$, es posible potenciar desde las diferencias la capacidad de adaptación de la empresa, como asimismo su potencialidad de innovación y creatividad.

Para lograr este objetivo, se propone el método de la negociación colaborativa ${ }^{107}$, destinado a orientar el proceso de diálogo entre las partes de la empresa extendida, como espacio necesario para las intervenciones en el lenguaje que permitan crear una nueva realidad ${ }^{108}$, desplazando el antiguo paradigma institucional a través de la autopoiesis ${ }^{109}$.

\section{HAX. Ibíd.}

102. ARDILES. Ibíd.

103. HAZEN. Ibíd.

104. HOEFSTEDE. Ibíd.

105. COX. Ibíd.

106. KORNBERGER. Ibíd.

107. MAJLUF. Ibíd.

108. ECHEVERRIA. Ibíd.

109. LIMONE, Aquiles y MARINOVIC, Milan: "The Theory of the Organization and the New Paradigms". En: Open Journal of Business and Management, 2013. Págs. 30 y ss.

Facultad de Derecho y Ciencias Sociales - Universidad de Valparaíso - Chile 
Asimismo, es importante recalcar la importancia de que las actividades anteriormente señaladas deben sumarse a acciones relevantes de coherencia con los planteamientos realizados a través del diálogo de la organización ${ }^{110}$.

Finalmente, el cambio planteado en este trabajo no busca generar una modificación procedimental dentro de la planificación de las organizaciones, sino que aportar al nuevo paradigma que resulta necesario ${ }^{111}$ para la sobrevivencia de las organizaciones.

\section{Bibliografía}

ALVES DE SOUZA, Filho: "La cuestión del sujeto individual y grupal en sociedades multiculturales en transformación". En: Revista Mexicana de Ciencias Políticas y Sociales, Vol. 50 N² 203, 2008. Págs. 53 y ss.

ARDILES, Jorge "De empresa y estrategia: Ideas y casos". En: Gráfica Metropolitana, 2012.

BARRET, Frank y COOPERRIDER, David: "Generative methaphor intervention: A new approach for working with systems divided by conflict and caught in defensive perception”. En: Journal of Applied Behavioral Sciene, Vol. $26 \mathrm{~N}^{\circ}$ 2, 1990. Págs. 219 y ss.

BLOCK, Fred y EVANS, Peter: "El Estado y la Economía". En: Peter Evans, Instituciones y desarrollo en la era de la globalización neoliberal", ILSA, 2007. Págs. 307 y ss.

BRAVO, Juan: “Análisis de Sistemas”. Evolución S.A. Santiago. 1998.

CALLON, Michel: "Some elements of a sociology of translation: domestication of the scallops and the fishermen of St Brieuc Bay”. En: The Sociological Review, VOL. 32 N 1, 1984. Págs. 196 y ss. CASTILLO, José: "Cambio social y enseñanza de administración de empresas”. En: Revista española de la opinión pública, N² 2, 1965. Págs. 109 y ss.

110. CLEGG. Ibíd.

111. LIMONE y MARINOVIC. Ibíd.

Revista de Ciencias Sociales - Número 68 (2016) - Universidad de Valparáíso - ISSN 0716-7725-Valparaíso, Chile 
CLEGG, Stewart, COUPASSON, David y PHILLIPS, Nelson: "Power and organizations". En: Fundations for Organizational Science,

Pine Forge Press, Londres, 2011.

COX, Taylor y BLAKE, Stacy: "Managing cultural diversity: implications for organizational competitiveness". En: The

Executive. Vol. $3 \mathrm{~N}^{\circ}$ 5, 1991. Págs. 45 y ss. Obtenido el 13 de marzo de 2016 de: http://www.jstor.org/stable/4165021

GARDELLA, Ma Mercè DARNACULLETA. "Derecho administrativo y autorregulación: La autorregulación regulada”. Obtenido el

15 de junio de 2014 de: http://www.tdx.cat/bitstream/handle/10803/

7681/tmdg.pdf? sequence $=7$

DE GEUS, Arie y GORIN, Jorge: "La empresa viviente". Ediciones Granica S.A. Buenos Aires. 2011.

DRUCKER, Peter. "La gerencia de Empresas". Ediciones Sudamericana. Buenos Aires, 2012.

ECHEVERRÍA, Rafael: "Ontología del Lenguaje” LOM Ediciones, Santiago. 2005 (Editor JC Sáez).

FREEMAN, R. Edward y Mc VEA, John: "A Stakeholder Approach to Strategic Management”. En: Working Paper $N^{\circ}$ 01-02: Virginia:

Darden Graduate School of Business Administration, University of Virginia. 2001.

FRIEDMANN, Reinhard: "Gestión y organización de empresas en el siglo XXI”. LOM. Santiago. 2004.

GRANOVETTER, Mark: "Economic action and social structure: the problem of embeddedness". En: American journal of sociology.

Chicago: University Chicago Press.Vol. $91 \mathrm{~N}^{\circ}$ 3, 1985. Págs. 481 y ss.

HAMEL, Gary: "Strategy as revolution". En: Harvard Business Review Obtenido el 8 de Junio de 2015 de: http://www. thegroupcreative. co.uk/wp-content/uploads/Strategy-as-revolution-Gary-Hamel.pdf

HAX, Arnoldo y MAJLUF, Nicolás: "Lecciones en estrategia: Hacia una gestión de excelencia". En: Ediciones UC. Santiago, 2014.

HAZEN, Mary Ann: "Towards polyphonic organization" En: Journal of Organizational Change Management, Vol. 6 N 5, 1993. Págs.15

y ss.

HITT, Micahel, IRELAND, R. Duane y HOSKISSON, Robert: "Administración Estratégica: Competitividad y globalización:

Conceptos y casos”. CENCAGE Learning. México. 2015.

Facultad de Derecho y Ciencias Sociales - Universidad de Valparaíso - Chile 
HOEFSTEDE, Geert: "Identifying organizational subcultures: An empirical approach". En: Journal of management studies, Vol. 35

$\mathrm{N}^{\circ}$ 1, 1998. Págs. 1 y ss. Obtenido el 8 de junio de 2015 de: http:// karhen.home.xs4all.nl/Papers/M\%26A/Hofstede\%20(1998).pdf

KENNY, Graham: "From a Stakeholder Viewpoint: Designing measurable objectives". En: Journal of Business Strategy. Vol. 33

$\mathrm{N}^{\circ}$ 6, 2012. Págs. 40 y ss.

KORNBERGER, Martin, STEWART R., Clegg y CARTER Chris: "Rethinking the polyphonic organization: Managing as discursive practice”. En: Scadinavian Journal of Management, Vol. 22 $\mathrm{N}^{\circ} 1$, 2006. Págs. 3 y ss.

LIMONE, Aquiles: "La empresa viviente: Más allá de la metáfora”. Enefa. 2004. Arica. Chile. Obtenido de Escuela de Comercio Pontificia Universidad Católica de Valparaíso.

LIMONE, Aquiles y MARINOVIC, Milan: "The Theory of the Organization and the New Paradigms". En: Open Journal of Business and Management, 2013. Págs. $30 \mathrm{y}$ ss.

LYOTARD, Jean Francois: "The Differend". En: University of Minessota Press. Vol. 46, 2014.

MAJLUF, Nicolás: "Los desafíos de la gestión: de lo formal a lo sutil". Aguilar Ediciones. Santiago. 2011.

MIGUÉLES, Miguel Martínez: "Validez y confiabilidad en la metodología cualitativa". En: Revista Paradigma. Vol. 27 N² 2, 2014. Págs. 1 y ss.

PENAGLIA, Francisco y VALENZUELA VAN TREEK, Esteban: "Rebeldía en Calama: desafío al orden centralista chileno en un contexto de boom minero". En: Revista Mexicana de Ciencias Políticas y Sociales, Vol. $59 \mathrm{~N}^{\circ} 222$, 2014. Págs. 161 y ss.

PORTER, Michael: "What is Strategy?". En: Harvard Business Review, Nov-Dic 1996. Págs. 61 y ss.

RADIO COOPERATIVA: "Agrosuper anunció el cierre indefinido de la planta en Freirina" 10 de de Diciembre de 2012 . Obtenido el 24 de julio de 2015 de: http://www.cooperativa.cl/noticias/pais/regionde-atacama/conflicto-en-freirina/agrosuper-anuncio-el-cierreindefinido-de-la-planta-en-freirina/2012-12-10/174949.html

RODRÍGUEZ, Darío y ARNOLD, Marcelo: "Sociedad y teoría de sistemas”. Editorial Universitaria. Santiago. Chile 1991.

Revista de Ciencias Sociales - Número 68 (2016) - Universidad de Valparáíso - ISSN 0716-7725-Valparáiso, Chile 
SENGE, Peter, ROBERTS, Charlotte, ROSS, Richard, SMITH Bryan, y KLEINER, Art: "La quinta disciplina en la práctica". Ediciones

Granica S.A. Buenos Aires. 2012.

TINAS, Jorge: "La gestión ambiental del: ecosistema urbano". En: Ábaco, $\mathrm{N}^{\circ}$ 3, Otoño 1987. Págs. 12 y ss.

TORRES, Danilo: "La nueva historia económica, la teoría de la regulación y el análisis histórico social". En: Apuntes del SENCES, Vol. $31 \mathrm{~N}^{\circ}$ 54, 2012. Págs. 61 y ss. 